\title{
INHIBITION AND ESCAPE OF SARS-COV TREATED WITH ANTISENSE MORPHOLINO OLIGOMERS
}

\author{
Benjamin W. Neuman, David A. Stein, Andrew D. Kroeker, \\ Hong M. Moulton, Richard K. Bestwick, Patrick L. Iversen, \\ and Michael J. Buchmeier*
}

\section{INTRODUCTION}

Identification of potential SARS-CoV antiviral compounds has progressed swiftly, thanks in part to the availability of bioinformatic and virus structural data. Antivirals that target the SARS-CoV superfamily 1 helicase and the 3C-related serine proteinase with low micromolar $\mathrm{EC}_{50}$ values have been reported. ${ }^{1-3}$ The papain-related cysteine proteinase may prove to be an unsuitable target, as a coronavirus molecular clone lacking one of the two known cleavage sites for this enzyme displayed only minor growth defects in cell culture. ${ }^{4}$ Other confirmed and putative viral enzymes including the polymerase, poly(U)specific endo-ribonuclease homolog, $S$-adenosyl-methionine-dependent ribose 2'-Omethyltransferase, and cyclic phosphodiesterase represent plausible anti-SARS targets. ${ }^{5}$ Antivirals targeting the interaction of the viral spike protein with the ACE-2 receptor, ${ }^{2,3,6}$ or with the spike-mediated fusion event, ${ }^{7-10}$ and showing micromolar-scale efficiency in cell culture, have been reported. Several groups have also reported antiviral in vitro efficacy with siRNAs. ${ }^{11}$

The antisense agents directed against single-stranded RNA are known to act by two general mechanisms: by causing damage to an RNA strand containing the complementary "target" sequence through priming of endogenous RNase $\mathrm{H}$ activity, or by stably binding to and steric interference with targeted RNA function. Phosphorodiamidate morpholino oligomers (PMO) act by the latter mechanism, duplexing to specific RNA sequence by Watson-Crick base pairing and forming a steric block. ${ }^{12}$ The most frequently successful targeting strategies for PMO-based gene knockdown involve interfering with translation initiation ${ }^{13}$ or masking splice sites. ${ }^{14} \mathrm{We}$ recently demonstrated antiviral effects in vitro for one peptide-conjugated PMO (P-PMO) complementary to the AUG translation start site region of a murine coronavirus replicase

\footnotetext{
* Benjamin W. Neuman, Michael J. Buchmeier, The Scripps Research Institute, La Jolla, California. David A. Stein, Andrew D. Kroeker, Hong M. Moulton, Richard K. Bestwick, Patrick L. Iverson, AVI Biopharma, Inc., Corvallis, Oregon.
} 
polyprotein. ${ }^{15}$ We reasoned that antiviral effects of P-PMO might be improved by choosing conserved RNA sequence elements and secondary structures critical for replication, transcription, and host factor interaction as targets. In this report, we demonstrate that antisense-mediated suppression of viral replication can be achieved by targeting conserved RNA elements required for viral RNA synthesis and translation.

\section{ANTIVIRAL P-PMO SELECTION AND EFFICACY}

PMO complementary to the genomic (positive-sense) strand, which were designed to bind regions identified by conservation or noted in the literature as critical for viral RNA synthesis, were synthesized (Fig. 1a). PMO were covalently linked to peptides $\mathrm{NH}_{2}$ RRRRRRRRRFFC-CONH ${ }_{2}$ or $\mathrm{NH}_{2}$-RRRRRFFRRRRC-CONH $\mathrm{C}_{2}$ designated as $\mathrm{R}_{9} \mathrm{~F}_{2}$ or $\mathrm{R}_{5} \mathrm{~F}_{2} \mathrm{R}_{4}$, respectively. Both types of peptide-conjugated $\mathrm{PMO}$ are henceforth referred to as $\mathrm{P}-\mathrm{PMO}$, and were used interchangeably in these studies. Cells treated with $\leq 20 \mu \mathrm{M}$ PMO were at least $80 \%$ viable, as measured by MTT viability assay (data not shown).

We tested P-PMO for correlates of antiviral efficacy: reduction of viral titer, rate of spread in vitro, and viral subgenomic RNA load. Vero-E6 cells were pretreated with PPMO $6 \mathrm{~h}$ prior to low multiplicity inoculation, and supernatants were collected for titration $24 \mathrm{~h}$ later (Fig. 1b). The most effective P-PMO (TRS2) decreased viral titers below the threshold of detection in the experiment shown, and reduced peak titers by 10,000-fold in some assays (not shown). TRS1 and TRS2 exhibited robust antiviral activity in the low micromolar range.

We next tested the effectiveness of P-PMO on viral persistence and spread of an established infection by performing plaque-size reduction assays. In this assay, cells were

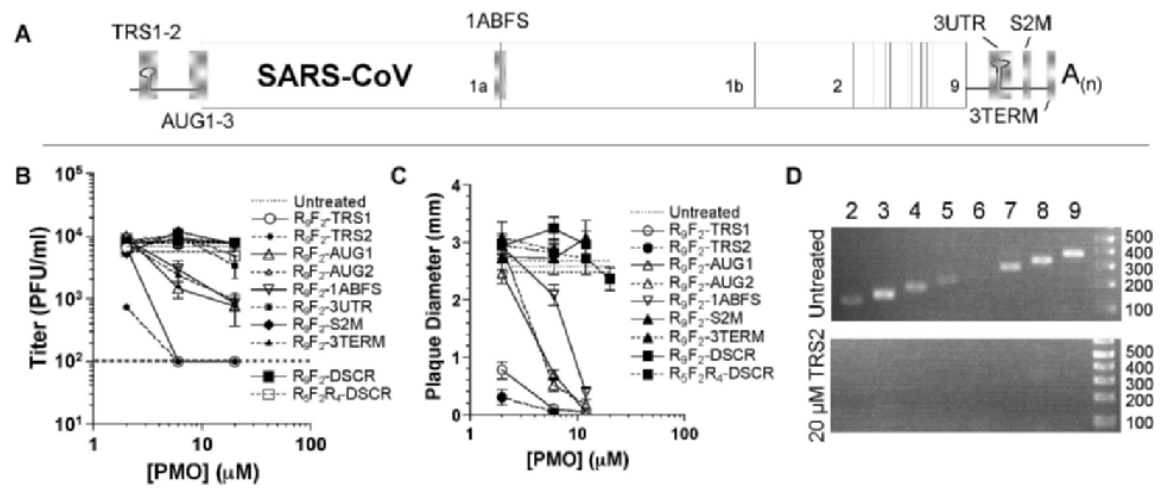

Figure 1. Binding sites and in vitro efficacy of MHV P-PMOs. A schematic presentation of the SARS-CoV genome shows the sites that were selected as P-PMO target sites (A). P-PMOs tested here included five designed to directly inhibit translation of the replicase open reading frame 1a (TRS1-2, AUG1-3), one to inhibit ribosomal frameshifting (1ABFS), three to bind conserved sequences in the 3'-untranslated region (3UTR, S2M, 3TERM), and one scrambled control sequence (DSCR). P-PMOs directed to the leader transcription regulatory sequence were most effective at reducing viral titer (B), and the diameter of viral plaques (C). Comparison of the detectable amounts of subgenomic RNAs present in infected cells (D). Amplicons specific to sgRNA 2-9 were obtained by RT-PCR on total RNA extracted from untreated or TRS2 P-PMO-treated cells. 
treated with P-PMO $1 \mathrm{~h}$ after inoculation with a standardized amount of SARS-CoV. Plaque diameter was measured $72 \mathrm{~h}$ after inoculation. The TRS2 P-PMO was most effective at reducing SARS-CoV spread in cell culture (Fig. 1C). We reasoned that inhibition of viral growth should correspond with a decrease in the viral RNA level, whether through inhibition of replicase expression, interference with discontinuous RNA synthesis at the leader TRS, or an alternate mechanism. Coronaviruses produce a nested set of sub-genome-length RNA species in infected cells. We investigated genomic and subgenomic RNA production $24 \mathrm{~h}$ after low-multiplicity inoculation. RT-PCR products specific to each of eight subgenomic RNA species were strongly amplified from untreated cells and cells treated with less effective P-PMO (Fig. 1D and data not shown). Equal volumes of RT-PCR products from an equivalent number of infected cells pretreated with $20 \mu \mathrm{M}$ TRS2 P-PMO were faint (i.e., sgRNA 8 and possibly 9) or undetectable (sgRNA 2-7; Fig. 1D). Genomic RNA synthesis was likewise qualitatively reduced by $20 \mu \mathrm{M}$ TRS2 P-PMO (data not shown).

\section{ESCAPE OF SARS-CoV AFTER SERIAL P-PMO SELECTION}

The error-prone replication of RNA viruses presents a rapid model for viral evolution and drug resistance studies. In order to assess the propensity for SARS-CoV to develop resistance to antisense P-PMO, a stock cultured from a plaque-purified biological clone of SARS-CoV was serially passaged on cells pretreated with P-PMO. Viral growth was assessed, after each passage (Fig. 2A). Treatment with $10 \mu \mathrm{M}$ TRS2 P-PMO strongly inhibited SARS-CoV growth for several passages. However, an increase in titer indicative of partial resistance was observed after seven passages. SARS-CoV plaque purified after 11 rounds of TRS2 P-PMO selection formed small plaques on Vero-E6 cells in the absence of P-PMO (Fig. 2B). TRS2 P-PMO-selected SARS-CoV displayed delayed growth kinetics compared with untreated SARS-CoV and other P-PMO-selected SARS-CoV (data not shown). RNA was isolated from plaque purified SARS-CoV selected after 11 rounds of serial P-PMO treatment. RT-PCR amplicons from 14 serially P-PMO-treated SARS-CoV were sequenced to determine whether the virus had undergone mutation during P-PMO selection. Three contiguous base changes of CTC to AAA at position 61-63, proximal to the leader TRS and within the target region of TRS2-P-PMO, appeared in only the 14 amplicons from TRS2-resistant SARS-CoV (Fig. 2C).

Thermal melting curve data for peptide-conjugated PMO/RNA duplexes with variable mismatches lead us to speculate that the three mutations at the TRS2-P-PMO target site reduce the effective melting temperature $(\mathrm{Tm})$ by $\sim 25-30^{\circ} \mathrm{C}(\mathrm{H}$. Moulton et al., manuscript in preparation and ${ }^{16}$ ). P-PMO binding affinity was compared using a reporter construct in which the luciferase reporter gene was placed immediately downstream of either the wild-type SARS-CoV TRS region or the same region with the CTC $\rightarrow$ AAA mutations observed in TRS2 P-PMO-selected SARS-CoV clones (Fig. 3A). TRS2 PPMO was approximately ten-fold less active against the 3-mismatch TRS target compared with the wild-type target $\left(\mathrm{EC}_{50}\right.$ of $500 \mathrm{nM}$ and $50 \mathrm{nM}$, respectively). The decreased sensitivity to TRS2 P-PMO was consistent with reduced P-PMO/target RNA duplex stability in partially-TRS2-resistant SARS-CoV. A similar observation was recently reported for HIV-1 escape variants resistant to siRNAs. ${ }^{17}$ 
A

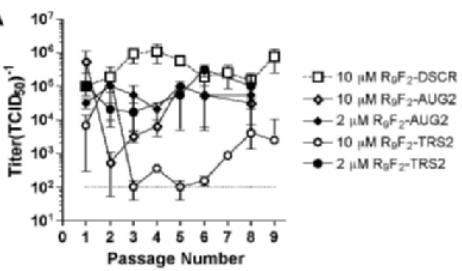

B

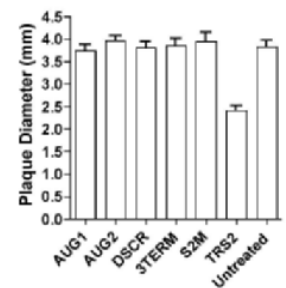

C

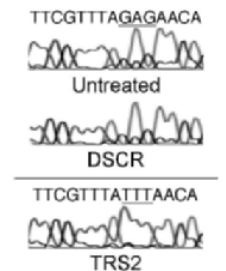

Figure 2. Escape of SARS-CoV after serial P-PMO passage. The titer of infectious SARS-CoV grown over nine rounds of treatment with selected P-PMOs was measured by TCID $_{50}$ titration (A). Error bars indicate standard error throughout. Plaque morphology of P-PMO-resistant SARS-CoV strains was assessed in the absence of P-PMO. Mean plaque diameter is shown (B). SARS-CoV strains selected after serial P-PMO treatment were sequenced. The region comprising the TRS2 P-PMO binding site is shown $(\mathrm{C})$. Sequences from untreated and DSCR-treated SARS-CoV represent wild-type sequence, and mutations found in all TRS2selected strains are underlined. Sequence is from the complementary (minus-sense) strand.

A

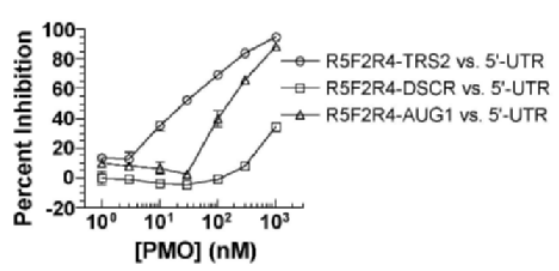

B 100

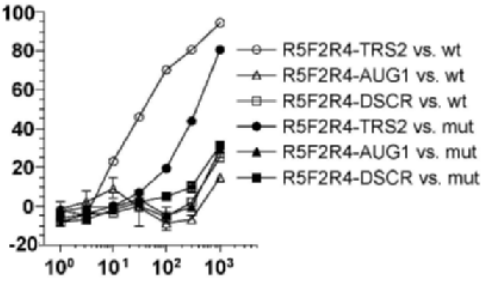

Figure 3. Mechanism of P-PMO escape and inhibition of SARS-CoV. Reporter gene expression from a synthetic RNA containing the SARS-CoV 5'-UTR upstream of the luciferase gene was measured in the presence of SARS-CoV P-PMO (A). Error bars represent standard error throughout. Reporter gene translation from a synthetic RNA containing the SARS-CoV 5'-untranslated region upstream of the luciferase gene (wt) or containing instead the mutated 5'-untranslated region found in serially TRS2-selected SARS-CoV upstream of the luciferase gene (mut) was measured in the presence of P-PMO (B).

The antiviral effects of TRS2 P-PMO were consistent with multiple mechanisms of action. In order to determine whether the high efficacy of TRS2 could be attributed to steric blockade of translation, a reporter construct was designed in which luciferase expression was initiated at the AUG codon of the authentic SARS-CoV replicase open reading frame 1a AUG, downstream of the SARS-CoV 5'-untranslated region. TRS2 PPMO was much more effective than AUG1 P-PMO in inhibiting reporter translation $\left(\mathrm{EC}_{50}\right.$ of $35 \mathrm{nM}$ vs. $185 \mathrm{nM}$; Fig, 3B). The TRS2 target site (bases 55-75) is sufficiently distal from both the 5'-terminus and the site of translation initiation to make it unlikely that interference with events of pre-initiation at the terminus (e.g., the $43 \mathrm{~S}$ complex loading onto mRNA) or initiation at the initiator AUG (e.g., 48S-complex formation and/or joining of $48 \mathrm{~S}$ and $60 \mathrm{~S}$ ribosomal subunits) forms the basis for the observed effect. We therefore concluded that TRS2 P-PMO primarily inhibits SARS-CoV growth by interference with translation, perhaps at the 43S-preinitiation complex scanning. 


\section{ACKNOWLEDGMENTS}

This work was supported by NIH grants AI059799, AI025913, NS41219 and by NIH/NIAID contract HHSN266200400058C. Figures and text adapted from Neuman et al., 2004. J. Virol. 79 (15):9665-9676. Used with permission.

\section{REFERENCES}

1. U. Bacha, J. Barrila, A. Velazquez-Campoy, S. A. Leavitt, and E. Freire, Identification of novel inhibitors of the SARS coronavirus main protease 3CLpro, Biochemistry 43, 4906-4912 (2004).

2. R. Y. Kao, W. H. Tsui, T. S. Lee, et al., Identification of novel small-molecule inhibitors of severe acute respiratory syndrome-associated coronavirus by chemical genetics, Chem. Biol. 11, 1293-1299 (2004).

3. C. Y. Wu, J. T. Jan, S. H. Ma, et al., Small molecules targeting severe acute respiratory syndrome human coronavirus, Proc. Natl. Acad. Sci. USA 101, 10012-10017 (2004).

4. M. R. Denison, B. Yount, S. M. Brockway, R. L. Graham, A. C. Sims, X. Lu, and R. S. Baric, Cleavage between replicase proteins p28 and p65 of mouse hepatitis virus is not required for virus replication, J. Virol. 78, 5957-5965 (2004).

5. E. J. Snijder, P. J. Bredenbeek, J. C. Dobbe,et al., Unique and conserved features of genome and proteome of SARS-coronavirus, an early split-off from the coronavirus group 2 lineage, J. Mol. Biol. 331, 991-1004 (2003).

6. L. Yi, Z. Li, K. Yuan, et al., Small molecules blocking the entry of severe acute respiratory syndrome coronavirus into host cells, J. Virol. 78, 11334-11339. (2004).

7. B. J. Bosch, B. E. Martina, R. Van Der Zee, et al., Severe acute respiratory syndrome coronavirus (SARS$\mathrm{CoV}$ ) infection inhibition using spike protein heptad repeat-derived peptides, Proc. Natl. Acad. Sci. USA 101, 8455-8460 (2004).

8. P. Ingallinella, E. Bianchi, M. Finotto, et al., Structural characterization of the fusion-active complex of severe acute respiratory syndrome (SARS) coronavirus, Proc. Natl. Acad. Sci. USA 101, 8709-8714 (2004).

9. S. Liu, G. Xiao, Y. Chen, et al., Interaction between heptad repeat 1 and 2 regions in spike protein of SARS associated coronavirus: implications for virus fusogenic mechanism and identification of fusion inhibitors, Lancet 363, 938-947 (2004).

10. K. Yuan, L. Yi, J. Chen, et al., Suppression of SARS-CoV entry by peptides corresponding to heptad regions on spike glycoprotein, Biochem. Biophys. Res. Commun. 319, 746-752 (2004).

11. Z. Wang, L. Ren, X. Zhao, T. Hung, A. Meng, J. Wang, and Y. G. Chen, Inhibition of severe acute respiratory syndrome virus replication by small interfering RNAs in mammalian cells, J. Virol. 78, 75237527 (2004)

12. J. Summerton, Morpholino antisense oligomers: the case for an RNase H-independent structural type, Biochim. Biophys. Acta 1489, 141-158 (1999).

13. A. Nasevicius and S. C. Ekker, Effective targeted gene 'knockdown' in zebrafish, Nat. Genet. 26, 216-220 (2000).

14. R. V. Giles, D. G. Spiller, R. E. Clark, and D. M. Tidd, Antisense morpholino oligonucleotide analog induces missplicing of C-myc mRNA, Antisense Nucleic Acid Drug Dev. 9, 213-220 (1999).

15. B. W. Neuman, D. A. Stein, A. D. Kroeker, et al., Antisense morpholino-oligomers directed against the 5' end of the genome inhibit coronavirus proliferation and growth, J. Virol. 78, 5891-5899 (2004).

16. D. Stein, E. Foster, S. B. Huang, D. Weller, and J. Summerton, A specificity comparison of four antisense types: morpholino, 2'-O-methyl RNA, DNA, and phosphorothioate DNA, Antisense Nucleic Acid Drug Dev. 7, 151-157 (1997).

17. E. M. Westerhout, M. Ooms, M. Vink, A. T. Das, and B. Berkhout, HIV-1 can escape from RNA interference by evolving an alternative structure in its RNA genome, Nucleic Acids Res. 33, 796-804 (2005). 